\title{
Diagnostic Evaluation of Anti-Tissue Transglutaminase and Anti- Endomysial Antibodies for Diagnosis of Coeliac Disease: Is There a Need for Duodenal Biopsy?
}

\author{
Jamal Golbahar ${ }^{1 *}$, Richard Brown ${ }^{2}$, Helen Melville ${ }^{1}$, Louise Messinger ${ }^{1}$, Morwenna Sampson ${ }^{1}$, \\ Timothy Watts ${ }^{1}$ and John O'Connor ${ }^{3}$
}

\begin{abstract}
${ }^{1}$ Department of Pathology, Clinical Biochemistry, Northern Devon NHS Trust, North Devon District Hospital, Barnstaple, UK; ${ }^{2}$ South West Pathology Services, Taunton, Somerset, UK; ${ }^{3}$ Department of Clinical Biochemistry, Royal Devon and Exeter Hospital, Exeter, UK
\end{abstract}

\begin{abstract}
Background and Objectives: Although duodenal biopsy is suggested as the gold standard method for diagnosis of coeliac disease (CD), high levels of immunoglobulin A anti-tissue transglutaminase (IgA anti-TTG) followed by positivity for immunoglobulin A anti-endomysial (IgA anti-EMA) have been used widely for the diagnosis. In this study, we tested the hypothesis that IgA antiTTG and IgA anti-EMA tests are useful for diagnosis of CD, without need for endoscopy and duodenal biopsy. Methods: CD diagnosis was made with $\operatorname{IgA}$ anti-TTG $>10 \mathrm{U} / \mathrm{mL}$ and $\operatorname{IgA}$ antiEMA positivity in presence of villous atrophy. Receiver operating characteristic (ROC) analysis was performed to establish the cut-off values of the IgA anti-TTG that could predict the presence of $\operatorname{IgA}$ anti-EMA positivity and villous atrophy from histology results. Results: The cut-off values for IgA anti-TTG that predict positivity for IgA anti-EMA (sensitivity: $89.7 \%$; specificity: $82 \%$; positive predictive value: $92.4 \%$ ) and villous atrophy (sensitivity: $88.9 \%$; specificity: $78.5 \%$; positive predictive value: $92.9 \%$ ) were 17 and $30 \mathrm{U} / \mathrm{mL}$, respectively. Conclusions: Serological testing can be used with high sensitivity and specificity for diagnosis of $\mathrm{CD}$, without biopsy and histology. This significantly improves the timeliness and effectiveness of $\mathrm{CD}$ diagnosis.
\end{abstract}

\section{Introduction}

Coeliac disease (CD) is a chronic gastrointestinal disorder and is the most common immuno-mediated enteropathy, reportedly affecting about $1 \%$ of the populations in Western countries. The purported causes of $\mathrm{CD}$ include both environmental (gluten) and genetic (human leucocyte antigen, DQ2/DQ8) factors. ${ }^{1}$ In CD, ingestion of gluten, a protein commonly found in wheat, rye and barley is believed to activate the immune response, with the con-

Keywords: IgA anti-TTG; IgA anti-EMA; Villous atrophy; Diagnostic; Evaluation. Abbreviations: CD, coeliac disease; IgA anti-TTG, immunoglobulin A anti-tissue transglutaminase; IgA anti-EMA, immunoglobulin A anti-endomysial; ROC, receiver operating characteristic.

Received: November 14, 2016; Revised: December 08, 2016; Accepted: December 16, 2016

DOI: 10.14218/ERHM.2016.00023

*Correspondence to: Jamal Golbahar, Department of Pathology, Clinical Biochemistry, Northern Devon NHS Trust, North Devon District Hospital, Barnstaple, EX31 4JB, UK. E-mail: jgolbahar@nhs.net sequence of intestinal damage and villous atrophy. In addition, CD is characterized by a variety of intestinal and extra-intestinal manifestations related to the subsequent deficiency of macronutrients and micronutrients related to the disease condition. ${ }^{2-4}$

The most frequently reported clinical signs and symptoms of classical CD include malabsorption (diarrhoea, steatorrhoea, unintentional weight loss) and of non-classical CD include anaemia, hepatic steatosis, osteopenia/osteoporosis and mouth ulcer. ${ }^{5}$ Untreated CD can lead to various complications that can severely affect quality of life, such as nutritional deficiencies, anaemia, osteoporosis and growth failure, as well as the possible development of other autoimmune disorders and malignancies. ${ }^{3-4}$ In current clinical practice, diagnosis of $\mathrm{CD}$ is normally carried out by first-line serology testing, with results of immunoglobulin A antitissue transglutaminase (IgA anti-TTG; high) and immunoglobulin A anti-endomysial (IgA anti-EMA; positive). Patients with CDindicative serology test results are then usually referred for duodenal biopsy to confirm the intestinal damage and villous atrophy. ${ }^{6-7}$

Although the duodenal biopsy and histology investigation has been considered as the gold standard for diagnosis of $\mathrm{CD}$, there are limitations to this approach; these include availability, unpleasantness, invasiveness, artefacts due to a non-longitudinal cut, poor specificity and high cost. ${ }^{8-9}$ For diagnosis of $\mathrm{CD}$, there are still questions and challenges remaining, particularly regarding who should be screened, how to screen, how to properly interpret the serologic test results and whether there is a need for further endoscopy, duodenal biopsy and/or histology investigation. In patients with suspected $\mathrm{CD}$, the IgA anti-TTG test is most commonly used as the first choice test to detect the presence of antibodies, but there are also questions about its accuracy. If there is a strong and ongoing clinical suspicion of $\mathrm{CD}$, IgA anti-EMA should be requested. However, there is still limited information regarding the sensitivity and specificity of IgA anti-TTG and IgA anti-EMA tests. A clearer understanding of the sensitivity and specificity of IgA anti-TTG and IgA anti-EMA will allow clinicians to better interpret test results and make a timelier and better informed diagnosis, ultimately supporting better administration of subsequent treatment. There are a number of studies suggesting that IgA anti-TTG levels are significantly and positively correlated with the severity of intestinal damage, but there are questions that remain as to whether there is a need for duodenal biopsies and histology investigations for all patients who present with positive serology results. ${ }^{10-15}$

In this study, we sought to determine the cut-off values for IgA anti-TTG to predict positivity for IgA anti-EMA and the presence of villous atrophy and we tested the hypothesis that high sensitivity and specificity of IgA anti-TTG and IgA anti-EMA would exclude 
Golbahar J. et al: Diagnostic Evaluation of Anti-Tissue Transglutaminase

the need for an endoscopy and histology procedures for diagnosis of $\mathrm{CD}$ in adults.

\section{Methods}

\section{Study population}

A total of 13,086 consecutive adult patients ( $>18$ years) referred from primary care from January 2012 to May 2016 with suspected $\mathrm{CD}$ according to presence of diarrhoea, steatorrhoea, unintentional weight loss, abdominal pain, bloating, anaemia, osteopenia/osteoporosis, hepatic steatosis, mouth ulcer and family history of CD were considered for study inclusion. All subjects were tested for the first time for IgA anti-TTG. Patients with IgA anti-TTG $>10 \mathrm{U} /$ $\mathrm{mL}$ were then tested for IgA anti-EMA; none of these patients were on the gluten-free diet. Patients with positivity for both IgA antiTTG and IgA anti-EMA underwent subsequent endoscopy with duodenal biopsies/histology. CD diagnosis was made according to the presence of villous atrophy associated with anti-TTG IgA $>10$ $\mathrm{U} / \mathrm{mL}$ and positivity for IgA anti-EMA. ${ }^{1}$

\section{Serology}

Serum anti-TTG IgA was analysed by immunoassay (Phadia 250; Thermo Fisher Scientific) and analysis of serum IgA anti-EMA was based on the fluorescein-labelled anti-human IgA (monkey oesophagus slides; BioSystems) and visualized with the aid of a fluorescence microscope, by immunofluorescence.

\section{Endoscopy and histology}

Multiple endoscopic duodenal biopsies were obtained by oesophagogastroduodenoscopy. Analysis of the duodenal biopsies was carried out at the Department of Histopathology. CD was diagnosed by pathological changes of the small intestine that included intraepithelial lymphocytosis, crypt hyperplasia and subtotal or total villous atrophy. ${ }^{9}$

\section{Statistical analysis}

Receiver operating characteristic (ROC) analysis was used to determine the cut-off values of IgA anti-TTG to predict positivity for IgA anti-EMA and villous atrophy by assessing the area under the curve (AUC) in addition to the sensitivity, specificity, positive predictive and negative predictive values. The ROC curves were constructed by plotting the sensitivity (true-positive) on the ordinate as a function of the complement of specificity (false-positive) for all possible cut-off values of the IgA anti-TTG test. Greater deviation towards the left upper corner with a high and significant AUC indicated good prediction of positive IgA anti-EMA and villous atrophy. All statistical inferences were made based on a twosided significant level of $P<0.05$ and were performed using IBM $^{\circledR}$ SPSS ${ }^{\circledR}$ Statistics version 21.0.

\section{Results}

Demographic characteristics, clinical symptoms, and serology and histology results of the patients with positivity for IgA anti-TTG are
Table 1. Biometric, serological, histological and clinical characteristics of patients with elevated IgA anti-TTG

\begin{tabular}{ll}
\hline Characteristic & $\begin{array}{l}\text { IgA anti-TTG }(>\mathbf{1 0} \mathbf{U} / \mathbf{m L}) \\
n=166\end{array}$ \\
\hline Age, years \pm SD & $50.7 \pm 12.4$ \\
Female, $n(\%)$ & $112(67.5)$ \\
Male, $n(\%)$ & $54(32.5)$ \\
Abdominal pain and & $42(25.3)$ \\
bloating, $n(\%)$ & \\
Anaemia, $n(\%)$ & $36(21.7)$ \\
Diarrhoea, $n(\%)$ & $37(22.3)$ \\
Weight loss, $n(\%)$ & $13(7.8)$ \\
Osteopenia/osteoporosis, $n(\%)$ & $15(9.0)$ \\
Diabetes, $n(\%)$ & $8(4.8)$ \\
Family History of CD, $n(\%)$ & $2(1.2)$ \\
Hepatic osteatitis,$n(\%)$ & $5(3.0)$ \\
Down's syndrome, $n(\%)$ & $2(1.2)$ \\
Mouth ulcer, $n(\%)$ & $6(3.6)$ \\
IgA anti-TTG, U/mL & $70.0 \pm 59.7$ \\
IGA anti-EMA-positive, $n(\%)$ & $136(82.0 \%)$ \\
Biopsy villous atrophy, $n(\%)$ & $142(85.5 \%)$ \\
\hline
\end{tabular}

shown in Table 1. During the study period, 13,086 subjects were referred for suspected CD and 166 were found to be IgA anti-TTGpositive $(1.3 \%)$. Out of those 166 patients, $136(82 \%)$ were $\operatorname{IgA}$ anti-EMA-positive and $142(85.5 \%)$ had villous atrophy. The mean IgA anti-TTG titre was $70.0 \pm 59.7 \mathrm{U} / \mathrm{mL}$. The prevalence of positive IgA anti-TTG was significantly higher in females (73.4\%) than males $(26.5 \%)$. The most common symptoms and conditions associated with positive IgA anti-TTG and CD were abdominal pain and bloating (26.5\%), anaemia (21.7\%) and diarrhoea (22.3\%).

ROC analysis showed that IgA anti-TTG with cut-off values of $17 \mathrm{U} / \mathrm{mL}$ (AUC=0.814 CI: $0.722-0.95, p=<0.001)$ predicts positivity for IgA anti-EMA (Fig. 1) with a high sensitivity (89.7\%), specificity $(82.7 \%)$, positive predictive value $(92.4 \%)$ and positive likelihood ratio $(2.6 ; 1.6-4.3, p=0.001)$, as shown in Table 2 . In addition, results from ROC analysis showed that $\operatorname{IgA}$ anti-TTG with a cut-off value of $30 \mathrm{U} / \mathrm{mL}$ (AUC $=0.715 \mathrm{CI}: 0.580-0.850, p=0.003$ ) predicts villous atrophy (Fig. 2) with a high sensitivity (88.9\%), specificity $(78.5 \%)$, positive predictive value $(92.9 \%)$ and positive likelihood ratio $(2.6 ; 1.0-4.1, p=0.01)$, as shown in Table 2.

\section{Discussion}

In this study, the diagnostic evaluation of serology tests for the diagnosis of $\mathrm{CD}$ was investigated and we also tested the hypothesis that serology tests are accurate and sensitive enough to allow for avoidance of intestinal biopsy and histology investigation with its limitations and cost. Histological examination is still considered as the gold standard method for diagnosis of CD in adults. However, there are limitations in endoscopy and histology procedures, including invasiveness, high costs and poor specificity in cases of duodenal lymphocytosis ${ }^{8}$, as some histological patterns are also seen in other clinical conditions. ${ }^{9}$ In this study, the major clinical 
Golbahar J. et al: Diagnostic Evaluation of Anti-Tissue Transglutaminase

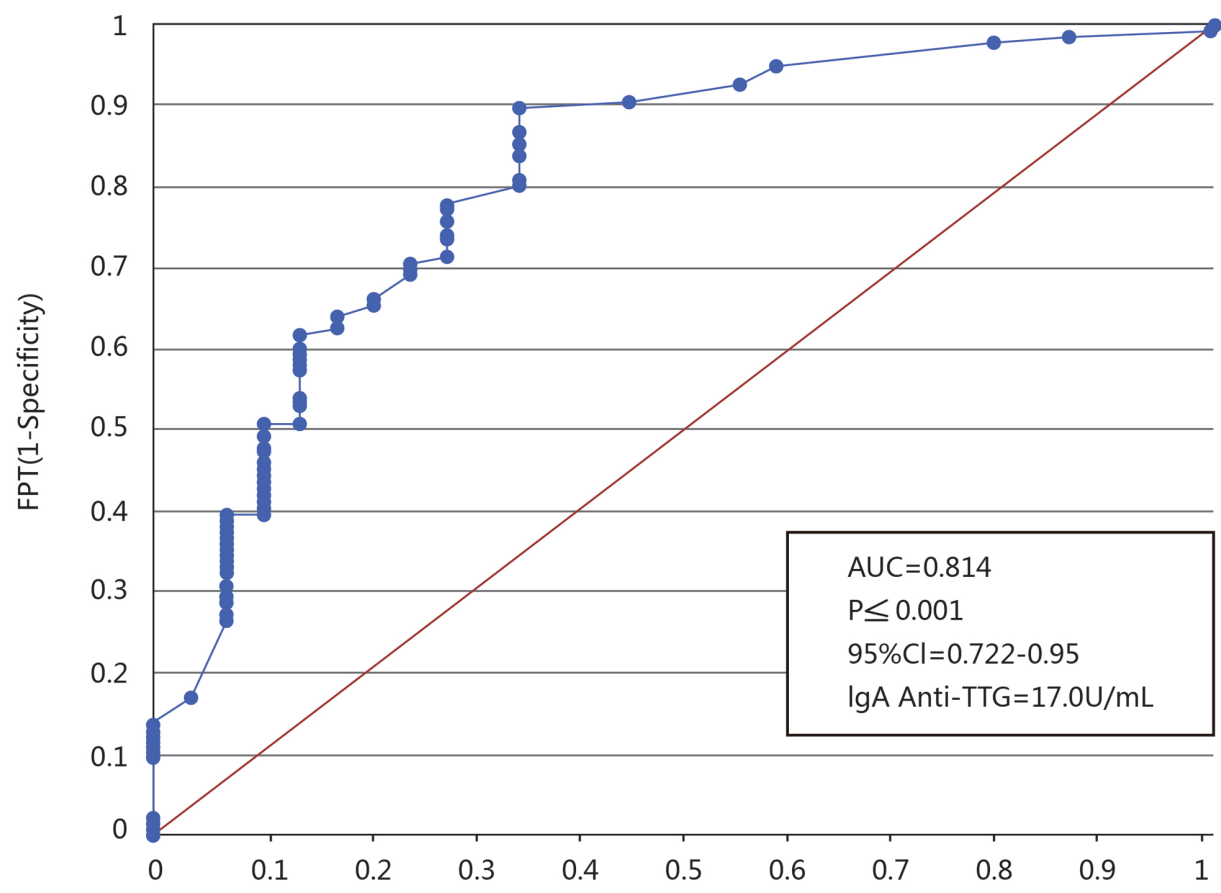

FPT(1-Specificity)

Fig. 1. ROC to determine the cut-off value of IgA anti-TTG for prediction of positivity for IgA anti-EMA.

conditions that were associated with positive IgA anti-TTG were abdominal pain, bloating, anaemia and diarrhoea, with other minor conditions recorded, including weight loss, osteoporosis/osteopenia, diabetes, hepatic osteatitis and mouth ulcer. There are some clinical conditions, such as liver disease, rheumatoid arthritis and inflammatory bowel disease, that may be associated with high IgA anti-TTG and can lead to false positive findings for CD. $.^{15} \mathrm{How}-$ ever, with positivity for IgA anti-EMA accompanied by symptoms and risk factors associated with $\mathrm{CD}$, it is more likely that the patients are suffering from $\mathrm{CD} .{ }^{16-17}$

ROC analysis showed that IgA anti-TTG levels at $17 \mathrm{U} / \mathrm{mL}$ predicted positivity for IgA anti-EMA, and at $30 \mathrm{U} / \mathrm{mL}$ predicted villous atrophy with high sensitivity, specificity and positive predictive values. Results from this study are consistent with the pre- vious studies that have suggested a strong relationship between IgA anti-TTG levels and small intestine histopathology and being highly specific for villous atrophy; thus, duodenal biopsy may not be required and may be avoided when IgA anti-TTG levels are 3-5 times greater than normal levels. ${ }^{13-14,18}$ This endoscopy-free diagnostic approach to CD could be particularly useful in situations where endoscopy is not available and where performing endoscopy would be very difficult, such as in patients who are elderly, pregnant, afflicted with Down's syndrome or mental illness. There are additional adult and children patients who may be unable or unwilling to undergo an endoscopy. Under these circumstances, assessment of the serological assay showing high IgA anti-TTG and positivity for IgA anti-EMA can have a supportive role.

This is the first retrospective study to investigate $\mathrm{CD}$ in adults in

Table 2. Diagnostic evaluation of IgA anti-TTG test for the prediction of positivity for IgA anti-EMA and villous atrophy

\begin{tabular}{lll}
\hline & IgA anti-EMA & Villous atrophy \\
\hline AUC & 0.814 & 0.715 \\
$95 \%$ CI & $0.722-0.95$ & $0.580-0.850$ \\
P & $<0.001$ & 0.003 \\
IgA anti-TTG, U/mL & 17.0 & 30.0 \\
Sensitivity & 89.7 & 88.9 \\
Specificity & 82.0 & 78.5 \\
Positive predictive value & 92.4 & 92.9 \\
Negative predictive value & 57.8 & 30.8 \\
Positive likelihood ratio, relative risk & $2.6(1.6-4.3)$ & $2.0(1.0-4.1)$ \\
Negative likelihood ratio & $0.16(0.09-0.28)$ & $0.35(0.19-0.64)$ \\
\hline
\end{tabular}


Golbahar J. et al: Diagnostic Evaluation of Anti-Tissue Transglutaminase

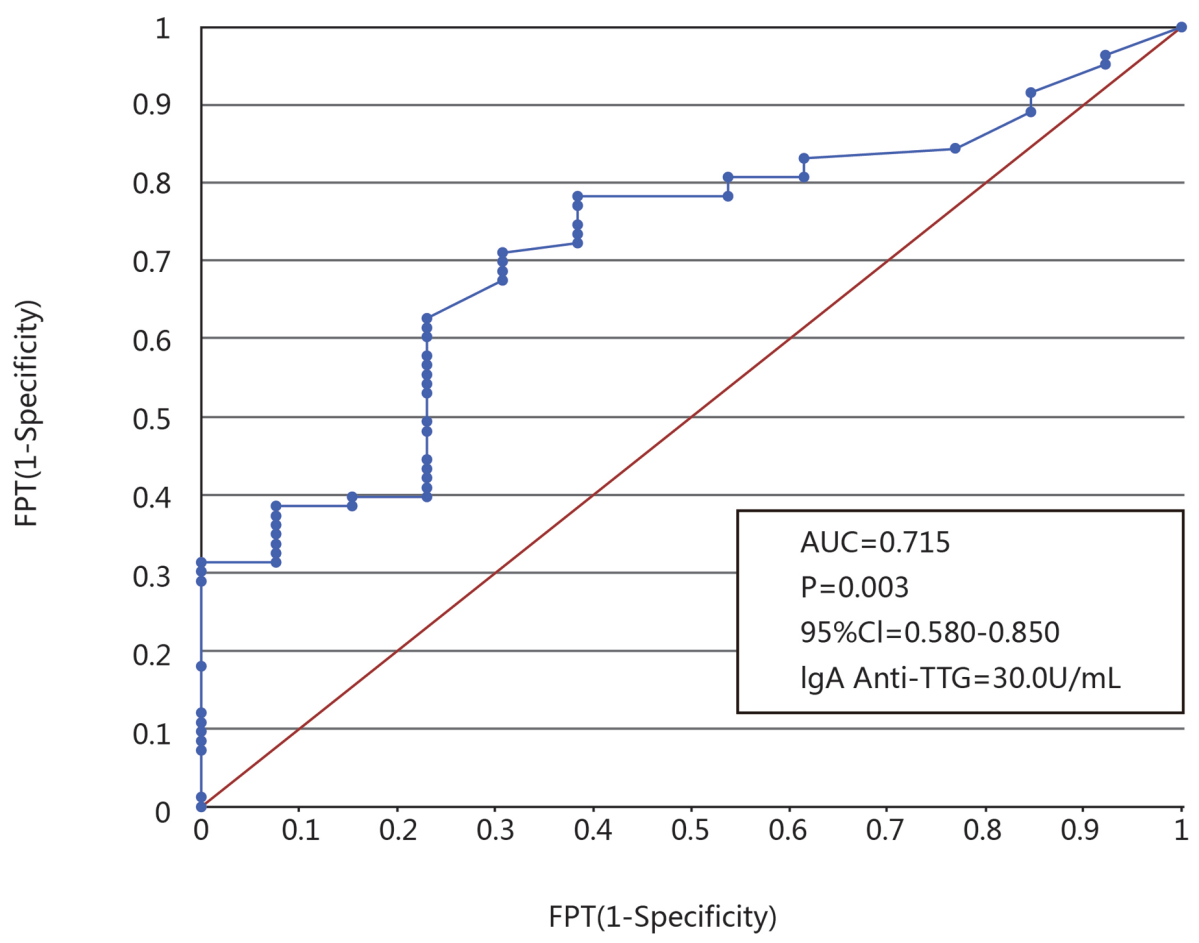

Fig. 2. ROC to determine the cut-off value of IgA anti-TTG for prediction of villous atrophy.

North Devon, UK. Results from this study suggest that the use of a diagnostic approach based on serology results showing IgA antiTTG levels of $17 \mathrm{U} / \mathrm{mL}$ to predict IgA anti-EMA and $30 \mathrm{U} / \mathrm{mL}$ to predict villous atrophy. Duodenal biopsy may not be required and could be avoided if IgA anti-TTG levels are 3-5 times greater than normal levels. This could have an impact on timely diagnosis of $\mathrm{CD}$ and significant reduction in CD diagnosis-related costs, as has been reported from previous studies and supports a cost-sparing and biopsy-free approach for diagnosis of CD. ${ }^{19}$

In conclusion, in adult patients with positivity for both IgA antiEMA and IgA anti-TTG, the latter at a level of $30 \mathrm{U} / \mathrm{mL}$, a diagnosis of $\mathrm{CD}$ could be reached without endoscopy and subsequent biopsy/histology. These results could contribute to improving the diagnostic work-up of CD for a significant reduction in diagnosisrelated costs.

\section{Acknowledgments}

The authors would like to acknowledge the Department of Histopathology at North Devon District Hospital, Barnstaple.

\section{Conflict of interest}

The authors have no conflict of interests related to this publication.

\section{Author contributions}

Acquisition of data (TW, HM, LM, RB, MS), analysis and interpretation of data $(\mathrm{JG}, \mathrm{JO})$, drafting of manuscript $(\mathrm{JG})$, critical revision (JO).

\section{References}

[1] Rubio-Tapia A, Hill ID, Kelly CP, Calderwood AH, Murray JA, American College of Gastroenterology. ACG clinical guidelines: diagnosis and management of celiac disease. Am J Gastroenterol 2013;108(5):656-676. doi:10.1038/ ajg.2013.79.

[2] James SP. National Institutes of Health consensus development conference statement on celiac disease, June 28-30, 2004. Gastroenterology 2005;128(4):S1S9. doi:10.1053/j.gastro.2005.02.007.

[3] Fasano A, Catassi C. Clinical practice. Celiac disease. N Engl J Med 2012;367(25):2419-2426. doi:10.1056/NEJMcp1113994.

[4] Kelly CP, Bai JC, Liu E, Leffler DA. Advances in diagnosis and management of celiac disease. Gastroenterology 2015;148(6):1175-1186. doi:10.1053/j.gastro.2015.01.044.

[5] Ludvigsson JF, Leffler DA, Bai JC, Biagi F, Fasano A, Green PH, et al. The Oslo definitions for coeliac disease and related terms. Gut 2013;62(1):43-52. doi:10.1136/gutjnl-2011-301346.

[6] Lewis NR, Scott BB. Systematic review: the use of serology to exclude or diagnosed coeliac disease (a comparison of the endomysial and tissue transglutaminase antibody tests). Aliment Pharmacol Ther 2006;24:47-54. doi:10.1111/ j.1365-2036.2006.02967.x.

[7] Gujral N, Freeman HJ, Thomson AB. Celiac disease: prevalence, diagnosis, pathogenesis and treatment. World J Gastroenterol 2012;42:6036-6059. doi:10.3748/wjg.v18.i42.6036.

[8] Salmi TT, Collin P, Reunala T, Mäki M, Kaukinen K. Diagnostic methods beyond conventional histology in coeliac disease diagnosis. Dig Liver Dis 2010;42(1):28-32. doi:10.1016/j.dld.2009.04.004.

[9] Tortora R, Capone P, Imperatore N, De Stefano G, Gerbino N, Leo M, et al. Predictive value of "Marsh 1" type histology in subjects with suspected celiac disease. Scand J Gastroenterol 2014;49(7):801-806. doi:10.3109/00365521.20 14.919019 .

[10] Zanini B, Magni A, Caselani F, Lanzarotto F, Carabellese N, Villanacci V, et al. High tissue-transglutaminase antibody level predicts small intestinal villous atrophy in adult patients at high risk of celiac disease. Dig Liver Dis 2012;44(4):280-285. doi:10.1016/j.dld.2011.10.013.

[11] Scoglio R, Di Pasquale G, Pagano G, Lucanto MC, Magazzù G, Sferlazzas C. Is intestinal biopsy always needed for diagnosis of celiac disease? Am J Gastroenterol 2003;98(6):1325-1331. doi:10.1111/j.1572-0241.2003.07455.x.

[12] Barker CC, Mitton C, Jevon G, Mock T. Can tissue transglutaminase antibody titers replace small-bowel biopsy to diagnose celiac disease in select pediatric populations? Pediatrics 2005;115(5):1341-1346. doi:10.1542/peds.2004-1392.

[13] Hill PG, Holmes GK. Coeliac disease: a biopsy is not always necessary for 
Golbahar J. et al: Diagnostic Evaluation of Anti-Tissue Transglutaminase

diagnosis. Aliment Pharmacol Ther 2008;27(7):572-577. doi:10.1111/j.13652036.2008.03609.x.

[14] Vivas S, Ruiz de Morales JG, Riestra S, Arias L, Fuentes D, Alvarez N, et al. Duodenal biopsy may be avoided when high transglutaminase antibody titers are present. World J Gastroenterol 2009;15(38):4775-4780. doi:10.3748/ WJG.15.4775.

[15] Bai JC, Fried M, Corazza GR, Schuppan D, Farthing M, Catassi C, et al. World Gastroenterology organisation global guidelines on celiac disease. J Clin Gastroenterol 2013;47(2):121-126. doi:10.1097/MCG.0b013e31827a6f83.

[16] Bizzaro N, Villalta D, Tonutti E, Doria A, Tampoia M, Bassetti D, et al. IgA and IgG tissue transglutaminase antibody prevalence and clinical significance in connective tissue diseases, inflammatory bowel disease, and primary biliary cirrhosis. Dig Dis Sci 2003;48(12):2360-2365. doi:10.1023/
B:DDAS.0000007875.72256.e8.

[17] Carroccio A, Di Prima L, Falci C, Le Moli C, Soresi M, Montalto G, et al. Predictive value of serological tests in the diagnosis of celiac disease. Ann Ital Med Int 2002;17(2):102-107.

[18] Dahlbom I, Korponay-Szabó IR, Kovács JB, Szalai Z, Mäki M, Hansson T. Prediction of clinical and mucosal severity of coeliac disease and dermatitis herpetiformis by quantification of $\operatorname{IgA} / \operatorname{IgG}$ serum antibodies to tissue transglutaminase. J Pediatr Gastroenterol Nutr 2010;50(2):140-146. doi:10.1097/ MPG.0b013e3181a81384.

[19] Cammarota G, Cesaro P, Martino A, Zuccalà G, Cianci R, Nista E, et al. High accuracy and cost-effectiveness of a biopsy-avoiding endoscopic approach in diagnosing coeliac disease. Aliment Pharmacol Ther 2006;23(1):61-69. doi:10.1111/j.1365-2036.2006.02732.x. 